'Servicio de Anestesiología y

Reanimación, Hospital Costa del Sol.

Marbella, España.

${ }^{2}$ Servicio de Anestesiología y

Reanimación, Hospital Puerta del Mar. Cádiz, España.

${ }^{3}$ Departamento de Anatomía Patológica, Biología Celular, Histología, Historia de la Ciencia, Medicina Legal y Forense y Toxicología, Facultad de Medicina Universidad de Cádiz. Cádiz, España.

${ }^{a}$ Magíster en Apoyo Respiratorio y Ventilación Mecánica.

bDoctorando en Ciencias de la Salud Universidad de Cádiz. Cádiz, España.

Trabajo no recibió financiamiento. Los autores declaran no tener conflictos de interés.

Recibido el 28 de junio de 2019 aceptado el 10 de junio de 2020 .

Correspondencia a: Pablo Romero Ávila Calle Ronda, número 19, $3^{\circ}$ C. Estepona (Málaga). España. Código Postal: 29680 deromeroyavila@gmail.com deromeroyavila@hotmail.com

\section{Historia de la ventilación mecánica. De la Antigüedad a Copenhague 1952}

\author{
PABLO ROMERO-ÁVILA ${ }^{1, \mathrm{a}, \mathrm{b}}$, CARLOS MÁRQUEZ-ESPINÓS $^{2, \mathrm{c}}$, \\ JUAN RAFAEL CABRERA-AFONSO ${ }^{3, \mathrm{c}}$
}

\section{The history of mechanical ventilation}

The aim of mechanical ventilation is to substitute physiological respiratory function. The boom of mechanical ventilation came during the XVIII century with the development of Reanimation Societies in Europe, who promoted the use of positive pressure ventilation modes. This type of ventilation caused new complications due to excessive positive pressure in the airway. Therefore, during the XIX century negative pressure ventilation predominated, which became essential during the second half of the $1^{\text {th }}$ century and first half of the $20^{\text {th }}$ century. Positive pressure ventilation was relegated to operating rooms until 1952, when it was imposed over negative pressure ventilation during the Copenhagen polio epidemic. Björn Ibsen contributed significantly to this change of ventilation paradigm, which led to the latest ventilation strategies and the development of the actual intensive care units.

(Rev Med Chile 2020; 148: 822-830)

Key words: Critical Care; History; Respiration, Artificial.
L

a ventilación mecánica $(\mathrm{VM})$ es una forma de ventilación artificial que emplea un aparato para suplir o colaborar en la respiración de una persona. Se utiliza en pacientes sometidos a anestesia general, en pacientes con alteraciones graves de la oxigenación, y en pacientes con alteraciones de la ventilación, ya sea de origen muscular, neurológico o respiratorio. Los orígenes de la VM son muy remotos. En este artículo vamos a describir su desarrollo histórico hasta 1952, fecha que marca el inicio de la ventilación mecánica tal y como la conocemos en la actualidad, tras un predominio de la ventilación mecánica de presión negativa. Asimismo, llegados a esta fecha, retrocederemos en el tiempo para analizar los factores que contribuyeron a la toma de decisión que puso fin a esta hegemonía.

\section{Antigüedad y Renacimiento: primeros antecedentes}

La importancia de la respiración para el mantenimiento de la vida es conocida desde la
Antigüedad. En Egipto, la respiración es referida como el aliento de la vida en el papiro de Ebers ${ }^{1}$. En Grecia, Hipócrates de Cos (460 a. C. - 370 a. C.) describió en su 'Tratado del aire' el primer intento documentado sobre la canulación orotraqueal: “... Se debe introducir una cánula dentro de la tráquea a lo largo de la mandíbula, entonces el aire debe ser guiado hacia los pulmones ${ }^{2}$...”.

En el Renacimiento, el médico suizo Paracelso (1493-1541) intentó reanimar a un paciente recién fallecido colocando un tubo en la boca, e insuflando aire a través de un fuelle en $1530^{3}$, mientras que Andrés Vesalio (1514-1564), anatomista belga, describió en De Humani Corporis fábrica VII (1543), que para mantener la vida de un animal había que realizar una traqueostomía e introducir un fragmento de caña, a modo de cánula, a través de la tráquea para insuflar aire ${ }^{4}$, manteniéndose de esta forma el latido cardiaco (Figura 1).

Durante el Barroco, un siglo más tarde y en plena Revolución Científica, el inglés Robert Hooke (1635-1703) repitió la experiencia de Vesalio en 


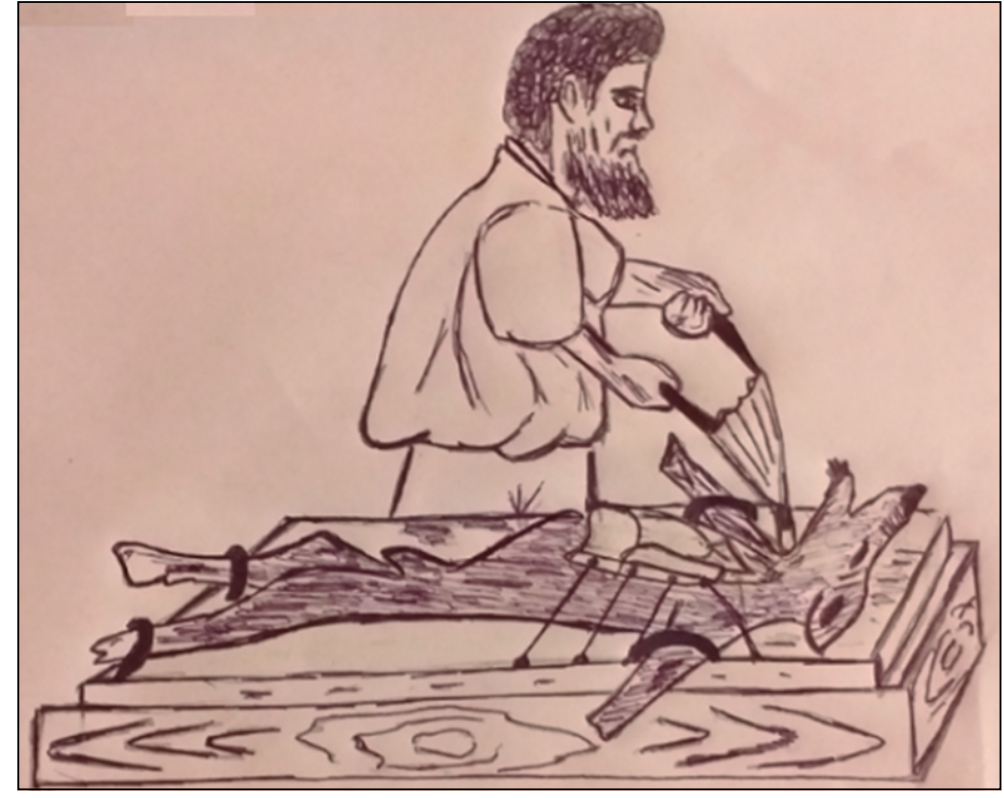

Figura 1. Representación de Vesalio ventilando a un perro a través de una caña insertada en la tráquea, con la ayuda de un fuelle. Los trabajos de este anatomista belga son considerados por muchos autores como el punto de partida de la ventilación mecánica. Dibujo original diseñado por el autor del artículo.
Londres, en la Royal Society. Para ello utilizó un perro como animal de experimentación. Este trabajo fue publicado en 1667, en la revista Philosophical Transactions, con el titulo "An account of an experiment made by $M$. Hook of Preserving Animals alive by Blowing trhough their lungs with Bellows".

Hasta este momento, las experiencias referidas en materia de VM pueden considerase fenómenos aislados, de carácter experimental en humanos o animales, y basados en la ventilación con presión positiva (VPP), es decir, a través de una presión mayor a la existente en la vía aérea.

\section{Siglo XVIII: auge de la Reanimación y desarrollo de la ventilación mecánica}

Durante el siglo XVIII, el ahogamiento se convirtió en un importante problema médico, y durante la segunda mitad del siglo se fundaron en Europa varias sociedades para promover la recuperación de las personas aparentemente ahogadas. La profundización en el conocimiento de la fisiología respiratoria contribuyó a que, en 1740, la Académie des Sciences de París aleccionara que la respiración boca a boca era el método más adecuado para la reanimación de estas personas. En esta época, la primera experiencia reportada de restablecimiento vital en humanos a través del boca a boca la hizo en 1744 el cirujano escocés William Tossach (1700-1771), relatando el éxito de esta maniobra en la asistencia a James Blair, un minero sofocado víctima de un incendio ${ }^{6,7}$.

Además, del boca a boca, en el s. XVIII se describieron también las primeras prácticas sobre la intubación endotraqueal en humanos. Benjamin Pugh (1715-1798), en 1754, y William Smellie (1697-1763), en 1763, relataron sus experiencias en resucitación neonatal mediante la canulación orotraqueal y posterior maniobra de ventilación boca-tubo. Más tarde, en 1772, John Fothergill (1712-1780) sustituyó la técnica de soplar el aire por la de emplear un fuelle $e^{8,9}$.

La experiencia de Fothergill con el empleo de fuelles le llevó a sugerir la posibilidad de lesión pulmonar como consecuencia de presiones elevadas, lo que hoy conocemos como barotraumatismo, ya que la fuerza ejercida por los fuelles no podía ser determinada. Por este motivo, recomendó el boca a boca como método preferencial de ventilación ${ }^{10}$.

Sin embargo, el boca a boca quedó relegado a un segundo plano tras el descubrimiento del dióxido de carbono y del oxígeno, en 1754 y 1774 respectivamente. Se postuló que el aire exhalado era deficiente en oxígeno, al haber sido procesado en los pulmones de otra persona, por lo que tomó más protagonismo la VPP con fuelles ${ }^{11}$. 
Con el auge de la Reanimación comenzaron a desarrollarse aparatos y sistemas para la VPP, como el sistema de doble vía inventado por el cirujano inglés John Hunter (1728-1793) en 1775 , modificado posteriormente por Charles Kite, quien incorporó unas válvulas de paso en los fuelles, y limitó el volumen de aire a $500 \mathrm{ml}$, muy cercano al volumen corriente ${ }^{12}$.

Otro aparato destacado fue el sistema de ventilación portátil diseñado por el obstetra francés François Chaussier (1746-1828), en 1780 (Figura 2). Dicho sistema estaba formado por una bolsa reservorio, y una mascarilla facial con sistema de sellado para compensar la pérdida de aire cuando se ventilaba con fuelles a través de la boca ${ }^{13}$.

En 1790, Han Courtois introdujo el sistema pistón-cilindro en sustitución de los fuelles. Este avance tecnológico fue muy bien acogido, y su uso se fue extendiendo ${ }^{12,13}$. No obstante, los avances en VPP se acompañaron de una serie de complicaciones asociadas, como el manejo inadecuado de las secreciones y las infecciones concomitantes ${ }^{3}$.

\section{Siglo XIX: ostracismo de la presión positiva}

Durante los primeros años del s. XIX, las dudas en cuanto a la seguridad de la VPP fueron aumentando, ya que con su uso aparecieron algunos casos de muerte por neumotórax. Estas dudas pasaron a ser evidencias con los trabajos de Jean Jacques Joseph Leroy d'Etiolles (1798-1860), en 1827, a quien se atribuye el descubrimiento del barotraumatismo producido por la VPP. Leroy demostró experimentalmente en animales que la VPP podía provocar la ruptura del alveolo, causando enfisema y neumotórax a tensión con fatales resultados ${ }^{13,14}$.

Los problemas relacionados con la VPP, y los avances en el conocimiento de la fisiología pulmonar, limitaron el progreso de este tipo de ventilación, centrándose las investigaciones científicas en el desarrollo de sistemas de ventilación de presión negativa (VPN), una modalidad primitiva de la Ventilación Mecánica No Invasiva (VMNI).

Estos sistemas se convirtieron en los dispositivos más importantes de VM durante el s. XIX. Su funcionamiento era similar en todos ellos: el cuerpo del paciente se colocaba dentro de una cámara más o menos hermética, con la cabeza

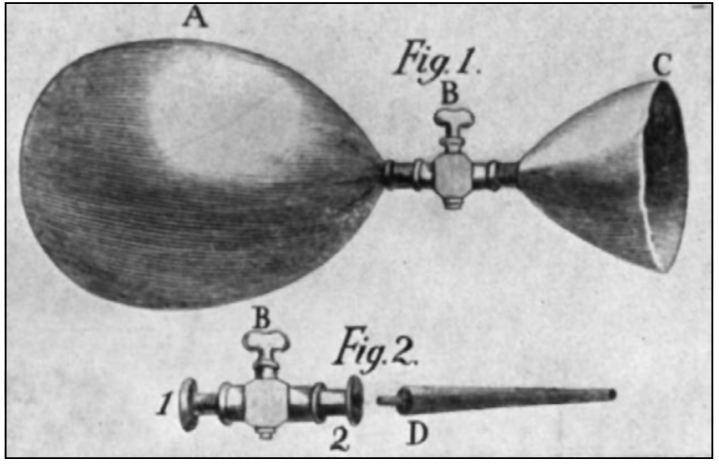

Figura 2. Sistema de ventilación portátil de Chaussier. En este caso, el componente para la ventilación es la bolsa reservorio. En su lugar se podía colocar un fuelle. Imagen tomada de Matioc AA. An Anesthesiologist's Perspective on the History of Basic Airway Management: The "Preanesthetic" Era-1700 to 1846. Anesthesiology 2016, volumen 124, número 2. Reproducido con permiso de Wolters Kluwer Health.

fuera de la misma, y en el interior de la cámara se aplicaba una presión negativa que provocaba la expansión del tórax. Posteriormente al retornar de nuevo la presión atmosférica se producía la espiración ${ }^{15}$.

La primera descripción de estos ventiladores de presión negativa fue la realizada por el médico escocés John Dalziel en 1838. En el ventilador de Dalziel el paciente se mantenía en posición sentada en su interior. La presión negativa era generada por unos fuelles que se encontraban en el interior de la caja, pero que se manejaban desde el exterior a través de un sistema de pistones y una válvula unidireccional ${ }^{16}$. Este tipo de ventiladores de cuerpo entero fueron conocidos como "ventiladores tanque" (Figura 3 ).

Posteriormente, en 1864, el estadounidense Alfred E. Jones patentó el primer "tank respirator" en América. El modelo era muy parecido al descrito por Dalziel. Una década más tarde, Eugène Woillez (1811-1882), médico francés, construyó un pulmón artificial al que llamó "Spirophore", presentándolo en París en 1876 (Figura 4). Este pulmón estaba compuesto por un cilindro donde se introducía al paciente acostado, con la cabeza fuera apoyada en un soporte. A nivel del cuello se colocaba un manguito de goma a modo de sellado para que el habitáculo quedara estanco. Se considera el prototipo de pulmón de acero $^{17}$. 


\section{Siglo XX: Impulso de la cirugía torácica y victoria en Copenhague}

Durante la primera mitad del s. XX continuó el predominio de la VPN, y se desarrollaron y perfeccionaron diferentes modelos y variantes.

Una de estas variantes fueron los respiradores de tipo coraza, los cuales cubrían únicamente la caja torácica. Entre ellos podemos destacar el biomotor de Eissenmenger, fabricado en 1904 y patentado en 1927 (prototipo en Figura 5). Sobre

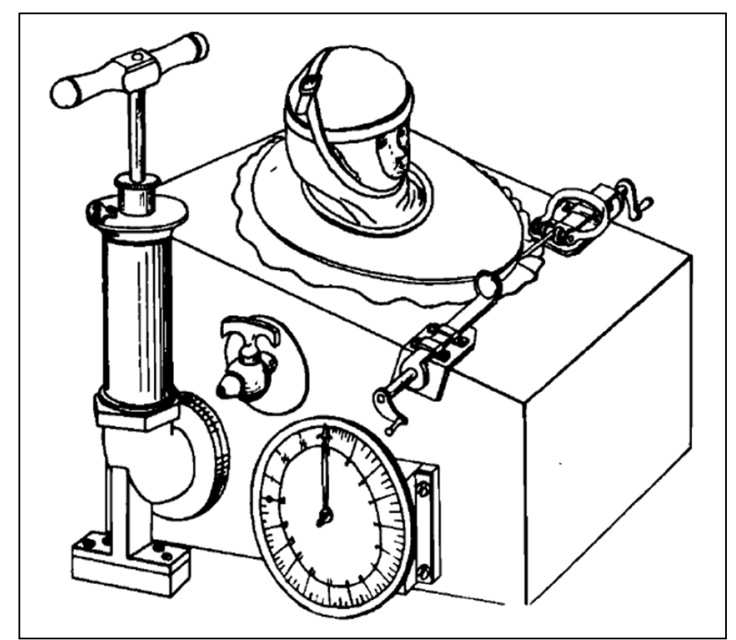

Figura 3. Ventilador de tipo tanque. El paciente se colocaba sentado en su interior con la cabeza fuera. Representación tomada de Woollam $\mathrm{CH}$. The development of apparatus for intermittent negative pressure respiration (1) 1832-1918. Anaesthesia 1976;31(4):537.47. Reproducido con permiso de Wiley and Sons. el biomotor se inspiraron otros modelos como el "Shalin-Stille cuirass" (1930), o el "Burstall Jacket" $(1937)^{18,19}$.

Otra variante fueron las cámaras de vacío o de presión negativa, como la presentada por Ernest Ferdinand Sauerbruch (1875-1951) en 1904, en el Congreso de la Sociedad Alemana de Cirugía, celebrado en Berlín ${ }^{20}$.

Pero, quizás, la variante más icónica y representativa de la VPN fueron los pulmones de acero. En 1928, el ingeniero Philip Drinker (1894-1972), y el fisiólogo Agussiz Shaw (1886-1940), desarrollaron el primer respirador de presión negativa para uso prolongado en Estados Unidos ${ }^{21}$. Este respirador, que pasó a conocerse como pulmón de acero, fue diseñado para poder ofrecer soporte ventilatorio a los pacientes afectados de poliomielitis (Figura 6). Más tarde, en 1931, Jack Emerson (1906-1997) construyó una versión del pulmón de acero más económica y eficiente, denominada Emerson Respirator ${ }^{22}$.

Los pulmones de acero y los respiradores de tipo coraza dominaron el ámbito de la Reanimación para las enfermedades respiratorias en la primera mitad del s. XX.

Durante la epidemia de poliomielitis de Copenhague de 1952, Björn Ibsen (1915-2007), un médico danés, convenció al jefe médico del "Blegdam Hospital”, Henri Cai Alexander Lassen (19001974), para realizar traqueostomía y ventilación manual con presión positiva, en los pacientes con afectación respiratoria. La idea resultó todo un éxito, y la mortalidad cayó espectacularmente de la noche a la mañana ${ }^{23}$. Pero ¿Qué llevó a Ibsen a

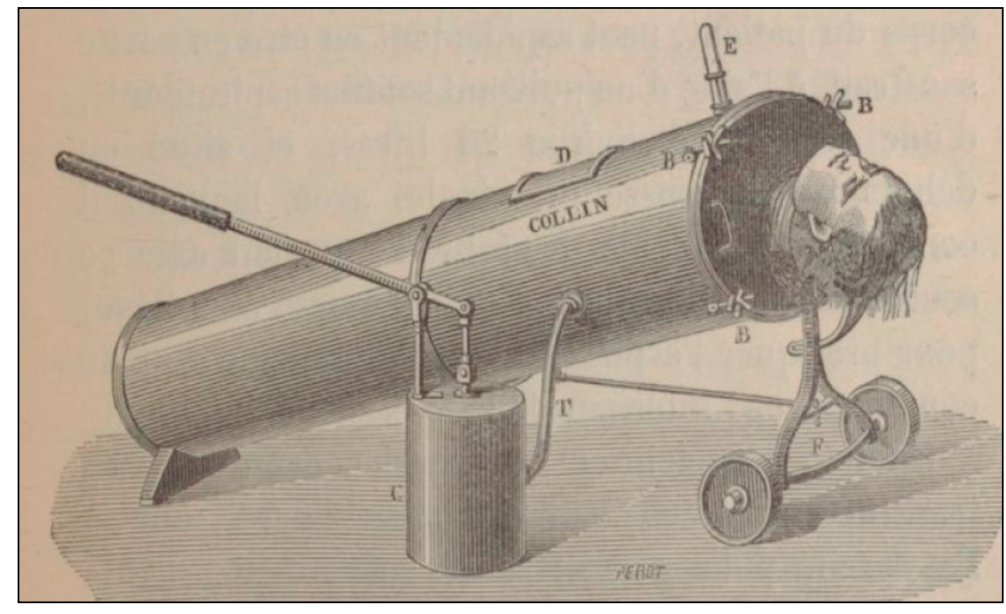

Figura 4. Spirophore de Woillez, París, 1876. El paciente se colocaba tumbado en el interior del tubo, con la cabeza fuera. Se considera el prototipo del pulmón de acero. Imagen disponible en: Woillez EJ. Du spirophore, appareil de sauvetage pour le traitement de l'asphyxie, et principalement de l'asphyxie des noyés et des nouveau-nés (communication à l'Académie de Médecine le 20 juin 1876): Bulletin de I'Académie de Médecine, Paris, tome V, 1876, 611-2. 
tener esta maravillosa idea? ¿Cómo se le ocurrió aplicar VPP, cuando la modalidad imperante en reanimación respiratoria era la VPN? Retrocedamos en el tiempo.

\section{Análisis de los factores que influyeron en el desarrollo de la VPP}

Con la introducción de la anestesia inhalatoria en 1846 por parte de W.T.G. Morton

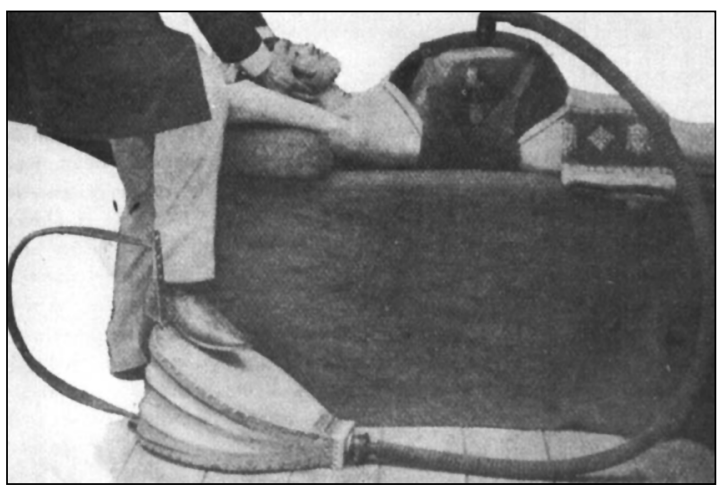

Figura 5. Prototipo del Biomotor de Eisenmmenger. La fuerza motriz es un fuelle de pie. En el Biomotor, el fuelle fue sustituido por un motor eléctrico. Reproducido de Woollam $\mathrm{CH}$. The development of apparatus for intermittent negative pressure respiration (1) 1832-1918. Anaesthesia 1976;31(4):537.47.
(1819-1868 $)^{24}$, la cirugía progresó a un ritmo vertiginoso, y comenzaron a aparecer las diferentes especialidades quirúrgicas. Sin embargo, la cirugía torácica presentaba un problema inherente: la apertura de la pleura provocaba el colapso del pulmón expuesto por neumotórax, dando lugar a la aparición de respiración paradójica y problemas hemodinámicos ${ }^{25}$.

Para solventar este problema, Sauerbruch construyó su cámara de presión negativa, dentro de la cual se podía llevar a cabo la cirugía de la cavidad torácica sin el colapso del pulmón (Figura 7). Los buenos resultados obtenidos hicieron que Sauerbruch viajara a EE. UU. y varios países europeos, para promocionar su cámara de presión diferencial. Sin embargo, esta cámara resultaba costosa y engorrosa ${ }^{20}$, y había otra corriente que tenía planteamientos opuestos para solucionar este problema.

Théodore-Marin Tuffier (1857-1929) Louis y Louis Hallion (1857-1929), dos cirujanos franceses, realizaron con éxito cirugías experimentales en perros bajo VPP e intubación laringotraqueal, por lo que propusieron en 1896 la insuflación de aire a través de la laringe o tráquea, para conseguir la distensión del pulmón ${ }^{26}$. Los resultados positivos en animales los animó a introducir la ventilación artificial intraoperatoria en humanos ${ }^{27}$, hecho que fue posible gracias al dominio que poseían tanto

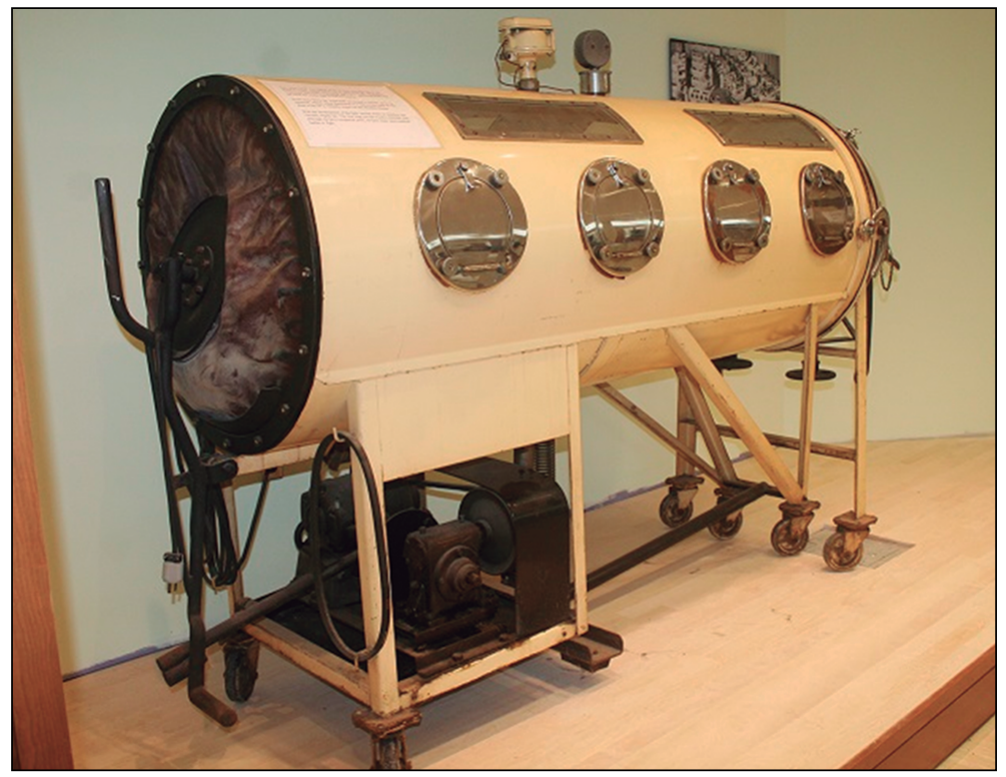

Figura 6. Pulmón de acero. Fuente: Colección de Historia de Enfermería "Josephine A. Dolan". Escuela de Enfermería de la Universidad de Connecticut. Con permiso. 

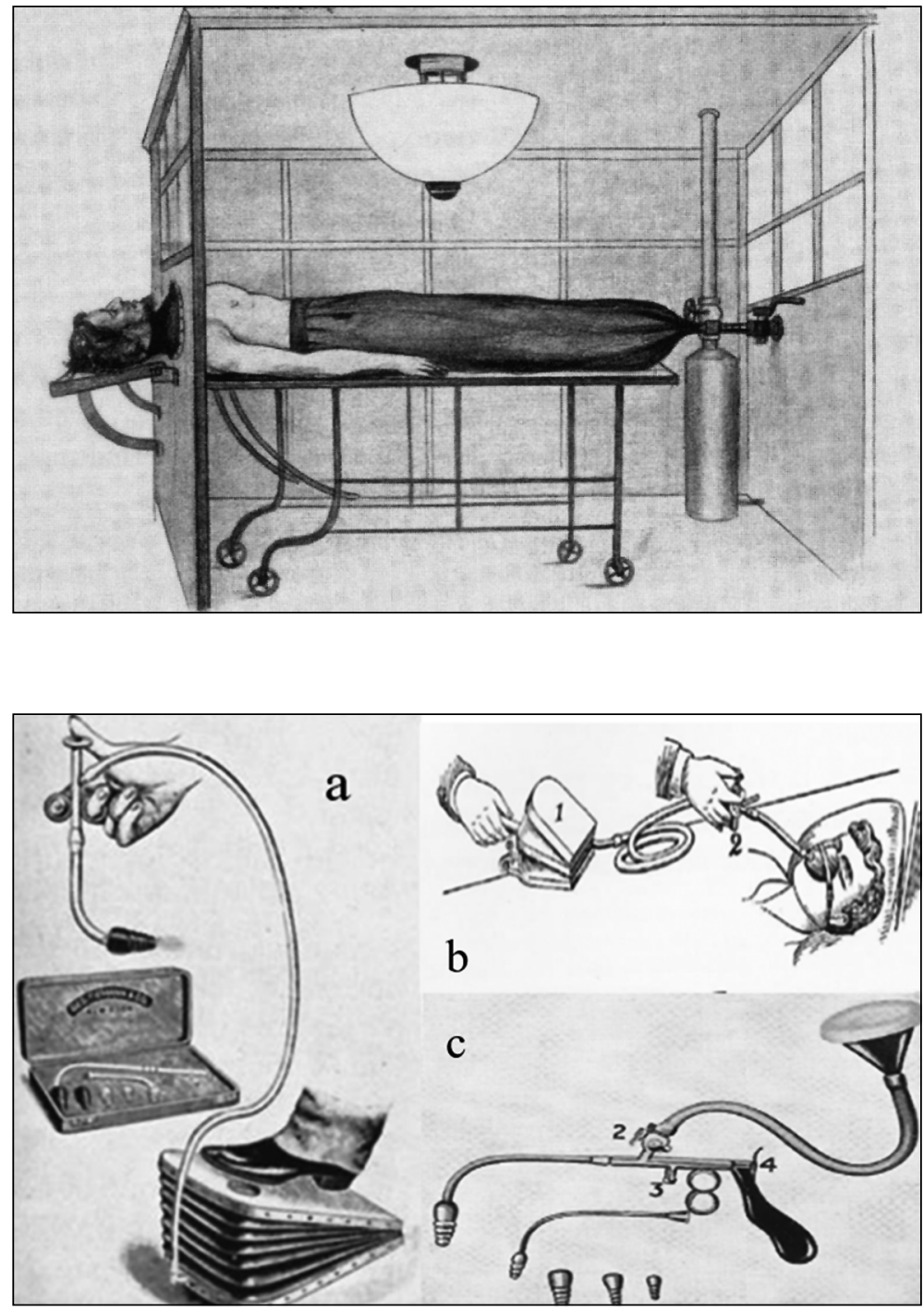

Figura 7. Cámara de presión diferencial de Sauerbruch. Permitió el desarrollo de la Cirugía Torácica. Presentaba como inconvenientes la necesidad de instalaciones costosas en un espacio destinado para tal fin, por lo que no era portable. Imagen de dominio público procedente de Wikimedia.
Figura 8. Aparato de Fell $O^{\prime}$ Dwyer para la ventilación laríngea de 1888 (a). Sistema original de George Fell para ventilación artificial con mascarilla (b) Modificación del aparato de Fell O 'Dwyer de Matas (1900). Imágenes reproducidas de Mushin WW, Rendell-Baker L. The principles of Thoracic Anaesthesia. Past and Present. 1953. Oxord. Blackwell Scientific Publications. Con permiso de Wiley and Sons. de la fisiología respiratoria, como de la regulación de la anestesia inhalatoria durante la ventilación $\operatorname{artificial}^{28}$.

Un año más tarde, Herbet M.N. Milton (18561921) publicó en la revista The Lancet un trabajo titulado Mediastinal Surgery, en el que ponía de manifiesto la necesidad de la VPP para el abordaje de la cavidad torácica, considerada como una "terra incógnita" 29 .

El potencial beneficio de la ventilación artificial en cirugía torácica fue vislumbrado también por Rudolph Matas (1860-1957), cirujano de Nueva Orleans de origen español. Matas estaba conven- cido de que, mediante el uso de la respiración artificial, la cirugía intratorácica podría lograrse con éxito. Esto llevó Matas, en colaboración con John Smythe, a diseñar un nuevo dispositivo basado en el aparato de Fell-O’Dwyer (Figura 8), con el que podía ventilar al paciente con VPP, y administrar oxígeno o cloroformo durante la respiración $\operatorname{artificial}^{30}$.

En la misma línea estuvieron trabajando los doctores neoyorquinos Natan W. Green (18711955) y Henry H. Janeway (1873-1921), quienes en 1910 destacaron la mejoría en las condiciones quirúrgicas en cirugía torácica bajo ventilación 
artificial, tras sus ensayos con animales ${ }^{31}$. Estos hechos fueron respaldados por Knut Harald Giertz (1876-1950) en 1916, quien demostró, por medio de la experimentación animal, la superioridad de la ventilación artificial por insuflación rítmica sobre el modelo de presión diferencial constante de Sauerbruch.

A partir de las observaciones de Giertz, en 1933 los cirujanos suecos Paul Frenckner y Clarence Crafoord (1899-1984), junto al ingeniero Anderson, diseñaron una máquina de anestesia que proporcionaba ventilación positiva intermitente conocida como el aparato de "Frenckner-Crafoord-Anderson". Este era el resultado de la combinación del Spiropulsator, un ventilador de presión positiva diseñado por Frenckner (Figura 9), con un aparato de anestesia de la compañía AGA. Crafoord lo empleó con éxito en varios centenares de pacientes sometidos a cirugía torácica mayor $^{32}$. A pesar de ello, todavía en 1937, Sauerbruch consideraba esta modalidad ventilatoria como peligrosa e innecesaria ${ }^{33}$.

Por otra parte, la introducción de la anestesia intravenosa con Tiopental, en 1934, y de los relajantes musculares en 1942, proporcionó mejores condiciones quirúrgicas, lo que favoreció la introducción de la ventilación artificial intraoperatoria en otras intervenciones más allá de la cirugía torácica ${ }^{34}$.

Trier Moerch (1908-1995), un médico danés que formaba parte de la resistencia durante la invasión alemana en la Segunda Guerra Mundial, aprendió a usar el Spiropulsator durante una estancia en Suecia, en 1943. De vuelta a su país, viendo que no podía importar este aparato por el bloqueo de la guerra, diseñó un respirador simi$\operatorname{lar}^{32,35}$. Después de la contienda, en 1949, viajó a EE. UU., donde rediseñó su ventilador y potenció el empleo de la VPP: fue el primer ventilador de este país en tener un fuelle en forma de concertina para leer el volumen corriente, y estaba disponible como una unidad separada, o como componente de varias máquinas de anestesia, como por ejemplo la máquina de Mckesson $^{32,36}$.

Ese mismo año, Ibsen se trasladó a Boston para especializarse en Anestesiología en el Hospital General de Massachusets, donde se familiarizó con la VPP. En 1950 regresó a Copenhague, donde comenzó a ejercer su nueva especialidad. Dos años más tarde, la ciudad se vio afectada por una de las peores epidemias de poliomielitis del

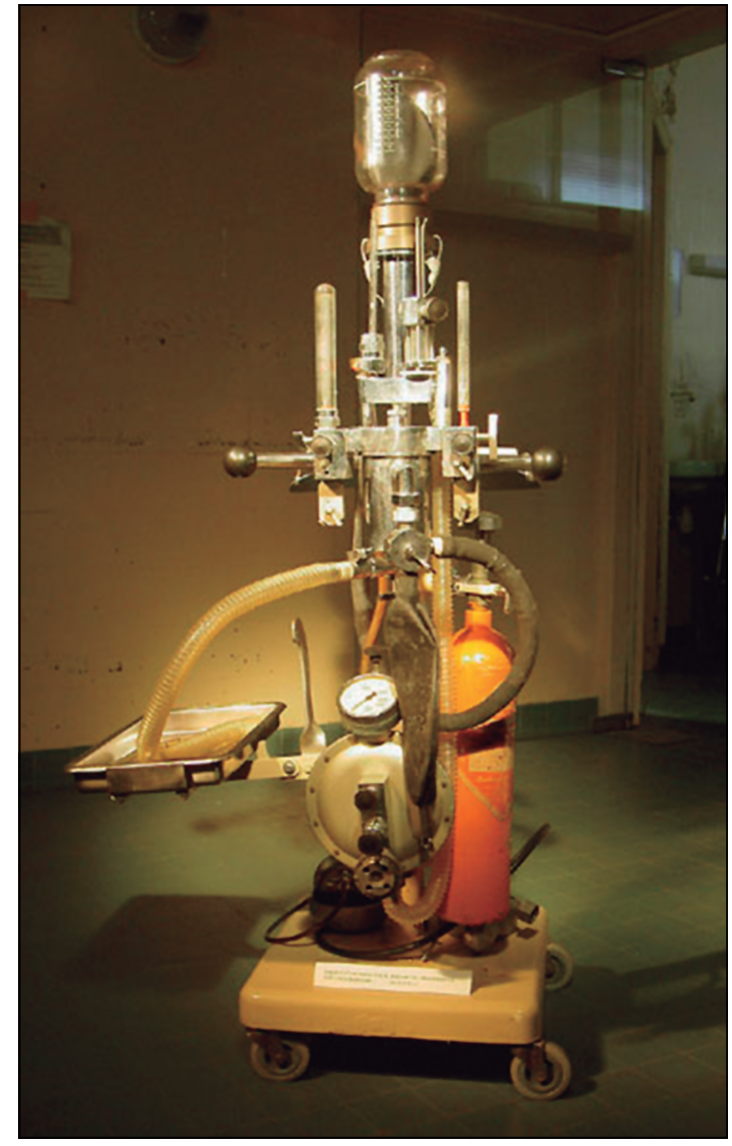

Figura 9. Spiropulsator de Frenckner. Fue uno de los primeros respiradores que se incorporaron a las máquinas de anestesia para proporcionar ventilación artificial. Fotografía del museo de anestesia del Hospital de Besançon, Francia. Reproducida con permiso.

mundo: más de cincuenta personas al día ingresaban en el Blegdam Hospital, algunos de ellos con afectación bulbar. La situación era desesperada, los pulmones de acero y los respiradores coraza funcionaban a pleno rendimiento, pero resultaban insuficientes ${ }^{37,38}$.

Ante este panorama, Lassen decidió llamar a Ibsen para conocer su punto de vista como anestesiólogo. Ibsen había aprendido en Boston que la retención de dióxido de carbono causaba hipertensión y sudoración, y reconoció estos síntomas en los pacientes de poliomielitis. Los pacientes estaban muriendo por insuficiencia respiratoria, y no por insuficiencia renal como se pensaba. Fue entonces cuando Ibsen propuso el 
tratamiento con VPP, y Lassen, que al principio se mostró escéptico, accedió cuando Ibsen demostró su eficacia en una niña de 12 años afectada de parálisis bulbar, y que se estaba ahogando con sus propias secreciones ${ }^{37,38}$.

Al comenzar a tratar a los pacientes con VPP, la mortalidad se redujo drásticamente, del $87 \%$ aproximadamente, al 40\%. Al final de la epidemia, alrededor de 1.500 estudiantes habían proporcionado ventilación manual durante unas 165.000 horas. Debido al desafío logístico que representó esta epidemia, se diseñaron las primeras Unidades de Cuidados Intensivos como las que conocemos en la actualidad ${ }^{39}$.

Este hecho determinó el triunfo de la VPP sobre la VPN, y marcó un antes y un después en la historia de la VM, y de la medicina en general. A partir de aquí se desarrollaron nuevas generaciones de ventiladores con tecnologías cada vez más sofisticadas, así como nuevas modalidades de ventilación, convirtiéndose la VM en uno de los pilares de los Cuidados Intensivos ${ }^{16}$; se implantó de forma definitiva la VPP a nivel intraoperatorio, lo que llevó a los distintos fabricantes a incorporar el ventilador en la máquina de anestesia ${ }^{40}$ $y$, por último, se convirtió en una herramienta multidisciplinar, sobre todo tras la introducción de la VMNI con presión positiva por Delaubier y Rideau en $1987^{41}$.

\section{Corolario}

La VM moderna tiene su punto de partida en 1952, al conseguir la VPP, durante la epidemia de poliomielitis de Copenhague, mejores resultados que la VPN en términos de morbimortalidad. Hasta ese momento, la VPN era la modalidad dominante como forma de ventilación artificial, ya que la VPP había quedado desprestigiada después de relacionarse con el barotraumatismo pulmonar. Sin embargo, múltiples factores contribuyeron a que la VPP se desarrollara en el ámbito quirúrgico. La cámara de Sauerbruch, diseñada para hacer frente al neumotórax asociado a la cirugía torácica, aunque ofrecía buenos resultados, precisaba de instalaciones que resultaban costosas y aparatosas. Varios estudios realizados en Francia, Estados Unidos y Suecia respaldaban el uso de la VPP en este tipo de cirugías. Además, la introducción en 1942 de los relajantes musculares en la práctica quirúrgica, favoreció la incorporación de la VPP en otro tipo de intervenciones. De esta forma, a inicios de la década de 1950, algunos anestesiólogos estaban familiarizados con el uso de la VPP. Björn Ibsen, que se había especializado en el Hospital General de Massachussets, formaba parte de esta escuela. Por este motivo, cuando se solicitó su ayuda en esta situación límite en la que los carísimos e insuficientes pulmones de acero eran incapaces de hacer frente a este desafío, su idea fue enseñar al resto de la comunidad médica de su hospital algo que él hacía habitualmente en las "salas de operaciones", y esta idea cambió la historia.

Agradecimientos: Los autores agradecen a los doctores García-López y Vivó Blasco su contribución en la elaboración del manuscrito, en la selección del material gráfico, así como en las labores de traducción.

\section{Referencias}

1. Rico FG, Botella M, Vargas L. Medicina y Teorías de la enfermedad en el Viejo Mundo. La antigüedad remota. Rev Inst Nac Enf Resp 2001; 14 (1): 178-95.

2. Rolando-Neri JZ. Historia de la ventilación mecánica. En: Cruz F, Fajardo G, Navarro FP, Carillo R, Editores. Ventilación mecánica. México D.F. México: Alfil; 2013.p.1-9.

3. Salas DA. Breve historia de la ventilación mecánica asistida. Acta académica 2000; 1: 89-91.

4. Vallejo-Manzur F, Perkins Y, Varon J, Baskett P. Andreas Vesalius, the concept of an artificial airway. Resuscitation 2003; 56: 3-7.

5. Baskett TF. Robert Hooke and the origins of artificial respiration. Resuscitation 2004; 60: 125-7.

6. Fitting JW. From breathing to respiration. Respiration 2015; 89 (1): 82-7.

7. Baker AB. Artificial respiration, the history of an idea. Med His 1971; 15 (4): 336-51.

8. Matioc A. An Anesthesiologist's Perspective on the History of Basic Airway Management. The "Preanectesic” Era -1700 to 1846. Anesthesiology 2016; 124 (2): 301-11.

9. O’Donnel CP, Gibson AT, Davis PG. Piching, electrocution, ravens'breaks, and positive pressure ventilation: a brief history of neonatal resuscitation. Arch Dis Child Fetal Neonatal Ed 2006; 91: 369-73.

10. Whitehead T, Slutsky AS. The pulmonary physician in 
critical care ${ }^{*} 7$ : ventilator induced lung injury. Thorax. 2002; 57 (7): 635-42.

11. Sternbach GL, Varon J, Fromm RE, Sicuro M, Baskett PJ. Galen and the origins of artificial ventilation, the arteries and the pulse. Resuscitation 2001; 49: 119-22.

12. Iglesias NR. Antecedentes históricos, conceptuales y contextuales sobre ventilación mecánica artificial y el proceso de destete. Mediciego 2011; 17 (1): 1-8.

13. Price JL. The evolution of breathing machines. Med Hist 1962; 6 (1): 67-72.

14. Leroy-d'Étiolles JJJ. Recherches sur l'asphyxie. Journal de physiologie 1827; 7: 45-65.

15. Yano R, Gonzalo JA, Fernández M. Historia de la ventilación mecánica. En González A, Gonzalo JA, Del Blanco A, Coordinadores. Manual de ventilación mecánica en medicina intensiva, anestesia y urgencias. Oviedo: Imprenta Gofer; 2005. p.1-9.

16. Kacmarek R. The Mechanical Ventilator: Past, Present, and Future. Resp Care 2011; 56 (8): 1170-80.

17. Woollam $\mathrm{CH}$. The development of apparatus for intermittent negative pressure respiration (1) 1832-1918. Anaesthesia 1976; 31 (4): 537-47.

18. Braun N. Negative Pressure Noninvasive Ventilation (NPNIV): History, Rationale, and Application. En Basner RC, Parthasarathy S, Editores. Nocturnal Non-Invasive Ventilation. Theroy, Evidence, and Clinical Practice. EE. UU.: Springer; 2015. p. 27-36.

19. Wollam $\mathrm{CH}$. The development of apparatus for intermittent negative pressure respiration (2) 1919-1976, with special reference to the development and uses of cuirass respirators. Anaesthesia 1976; 31 (5): 666-85.

20. Cherian SM, Nicks R, Lord RS. Ernest Ferdinand Sauerbruch: Rise and Fall of the Piooner of Thoracic Surgery. World J Surg 2001; 25 (8): 1012-20.

21. Drinker P, Shaw LA. An apparatus for the prolonged administration of artificial respiration: I. A Design for Adults and Children. J Clin Invest 1929; 7 (2): 229-47.

22. Bause GS. Emerson Respirator or “iron lung”. Anesthesiology 2009; 110 (4): 812.

23. West JB. The physiological challenges of the 1952 Copenhagen poliomyelitis epidemic and a renaissance in clinical respiratory physiology. J Appl Physiol 2005; 99 (2): 424-32.

24. Duncum BM. The development of inhalation anaesthesia. London: Oxford University Press; 1947. p.131-4.

25. Mushin WW, Rendell-Baker L. The principles of Thoracic Anaesthesia. Past and Present. Oxord: Blackwell
Scientific Publications; 1953.

26. Tuffier T, Hallion L. Respiration artificielle par insufflations pulmonaire dans certaines operations intrathoraciques. Gaz Hebd Med Chir 1896; 43: 1131.

27. Tuffier T, Hallion L. Operations intrathoraciques avec respiration artificielle par insufflation. C R Soc BioI 1896; 48: 951-4.

28. Tuffier T, Hallion L. Sur la régulation de la pression intra-broncrique et de la narcose dans la respiration artificielle par insufflation. C R Soc BioI 1896; 48: 1086-8.

29. Milton H. Mediastinal Surgery. Lancet 1897; 1: 872-5.

30. Matas R. Artificial respiration by direct intralaryngeal intubation with a modified O'Dwyer tube and a new graduated air-pump, in its applications to medical and surgical practice. Am Med 1902; 1-2.

31. Green NW, Janeway HH. Artificial respiration and intrathoracic oesophageal surgery. Ann Surg 1910; 52: 58-66.

32. Moerch ET. Controlled Respiration by Means of Special Automatic Machines as Used in Sweden and Denmark. Anaesthesia 1948; 3: 4-11.

33. Brodsky JB, Lemmens HJ. The history of anesthesia for thoracic surgery. Minerva Anestesiol 2007; 73 (10): 51324.

34. Sykes K, Bunker J. Curare tranforms anesthesia. En Sykes K, Bunter J, editores. Anaesthesia and the Practice of Medicine: Historical Perspectives. London: Hodder \& Stoughton. 2011. p.137-52.

35. Somerson JS, Sicilia MR. Historical perspectives on the development and use of mechanical ventilation. AANA J 1992; 60 (1): 83-94.

36. Rosenberg H, Axelrod JK. Ernst Trier Mørch: Inventor, Medical Pioneer, Heroic Freedom Fighter. Anesth Analg 2000; 90: 218-21.

37. Ibsen B. The anaesthetist's viewpoint on the treatment of respiratory complications in poliomyelitis during the epidemic in Copenhagen, 1952. Proc R Soc Med 1954; 47 (1): 72-4.

38. Richmond C. Bjørn Ibsen. BMJ 2007; 335 (7621): 674.

39. Slutsky AS. History of Mechanical Ventilation. From Vesalius to Ventilator-induced Lung injury. Am J Respir Crit Care Med 2015; 191 (10): 1106-15.

40. Wilkinson DJ. Evolution of the Anesthesia Machine. Curr Anaesth Crit Care 1991; 2 (1): 51-6.

41. Díaz-Lobato S, Sagrario-Mayoralas A. La ventilación mecánica no invasiva moderna cumple 25 años. Arch Bronconeumol 2013; 49 (11): 475-9. 